\title{
Immunohistochemical expression of apoptotic factors, cytokeratins, and metalloproteinase-9 in periapical and epithelialized gingival lesions
}

\author{
Angelo Leone', Maria Laura Uzzo', Francesca Rappa', Inaya Abdallah Hajj Hussein², \\ Aldo Gerbino1, Giovanni Francesco Spatola ${ }^{1}$, Abdo Jurjus ${ }^{2}$ \\ ${ }^{1}$ BioNec, Section of Histology and Embryology, Palermo University Medical and Dental School, \\ Palermo, Italy \\ ${ }^{2}$ Department of Anatomy, Cell Biology and Physiology, American University of Beirut, Beirut, Lebanon
}

\begin{abstract}
The aim of the study was to assess the involvement of apoptotic factors, cytokeratins and metalloproteinase-9 in the histogenesis of both Epithelialized Gingival Lesions (EGL) and Periapical Lesions (PAL). 55 consecutive patients, 30 with PAL and 25 with EGL, were selected for the study after clinical and radiological examinations. The PAL patients had severe periapical lesions and tooth decay with exposure of the pulp chamber. All PAL and EGL biopsies were surgically extracted, fixed in 10\% buffered formalin, and processed for routine light microscopy. Ten biopsies of each category were processed for immunohistochemistry (IHC). Serial paraffin sections were stained by IHC with appropriate antibodies to detect cytokeratins (CKs) 1, 5, 8, 10 and 14, caspase-3 and -9, metalloproteinase-9, and for PCNA and TUNEL assays. Both PAL and EGL showed a high expression of the cytokeratin 1, 5 and 8 with higher expression in EGL. Moreover, CK10 was markedly less intense expressed in EGL compared to PAL, while CK14 was almost three times stronger expressed in EGL. The expression of caspase-3 and -9 was stronger in PAL compared to EGL, however, the difference was only significant for caspase-9. In PAL apoptosis detected by TUNNEL method and the expression of MMP-9 were higher than in EGL, whereas PCNA was significantly more expressed in EGL. The results clearly suggest that both lesions have exclusively an epithelial origin and that epithelial proliferation was correlated with the degree of apoptosis in both entities. PAL and EGL presented mostly similar cytokeratin expression except for CK10 and CK14, though with marked differences in the distribution and intensity of IHC reactions. Finally, the degradation of extracellular matrix in both lesions could be partially attributed to the strong presence of MMP-9. (Folia Histochemica et Cytobiologica 2012, Vol. 50, No. 4, 497-503)
\end{abstract}

Key words: cytokeratins, MMP-9, caspase-3, caspase-9, perapical lesions, epithelial gingival lesions, apoptosis, IHC, PCNA, TUNEL

\section{Introduction}

Periapical lesions (PAL) are infections around the root of a tooth, resulting usually from spreading of dental caries. They extend into nearby bone, causing osteo-

Correspondence address: A. Leone,

BioNec. Sezione di Istologia ed Embriologia, Facoltà di Medicina e Chirurgia, Via Del Vespro, 12990127 Palermo; tel. +39 09165535 81, fax: +39 09165535 86; e-mail: angelo.leone@unipa.it myelitis; or, more often, spread to soft tissues, causing cellulitis and a swollen face. The presence of epithelium has been often demonstrated in periapical lesions, originating mostly from the epithelial residues of Malassez [1]. Histologically, PAL appears as a cyst rich with inflammatory cells and surrounded by a thin layer of epithelial cells [2].

On the other hand, epithelialized gingival lesions (EGL) are specific inflammatory damages which often lead to a increase of gum volume. They may have a chronic course, with increased gum volume, or an acute course with abscess, which can occur with an 
exacerbation of the chronic inflammation [3]. In general, EGL represents many types of gingival lesions. This work focused on the topography of the lesion rather than the differential diagnosis.

Apoptotic mechanisms are responsible for tissue homeostasis and are implicated in inflammatory processes of marginal periodontal lesions as well as other pathological changes. However, the exact role of apoptosis in PAL and EGL remains unclear [4-6]. Apoptosis is considered as a genetically regulated process activated by a variety of stress stimuli, including physical and mechanical ones [5-8]. Caspase-9, the initiator caspase, is induced by a number of stress signaling pathways that cause release of cytochrome $\mathrm{c}$ from mitochondria and activation of apoptosome that in turn cleaves the pro-enzyme of caspase-9 into the active form. The latter is able to activate other pro-caspases, like pro-caspase-3, which inactivates several cellular targets, like poly-ADP-ribose polymerase, and induces DNA cleavage [9]. Several stressors (e.g., hypoxia, bacteria, etc) induce mitochondrial release not only of cytochrome c [10], but also of Hsp60 [11, 12]. The latter accelerates pro-caspase3 cleavage and apoptosis activation [13-15].

Since the growth rate or activity of inflammatory lesions depends on the balance between cell proliferation and death, we examined immunoreactivity for PCNA antigen as a cell proliferation marker [2], a sensitive indicator detectable during active DNA synthesis or cell division [16]. An imbalance between cell proliferation and cell death may possibly determine some pathologies [17]. On the other hand, cytokeratins constitute the principal component of the cytoskeleton in epithelial cells. Their expression of CKs is often organ- or tissue-specific [18, 19]. Cytokeratins' expression is being used for the diagnosis of different lesions and as reliable marker for epithelial differentiation [20]. The visualisation of cytokeratins shows the location of epithelium in apical lesions, and consequently enables differentiation between cysts and epithelialized granulomas, as well as the determination of the histological type and prognosis [18-22]. Cytokeratins are classified, based on their molecular weight, in 2 subfamilies, the first comprises CKs from 1 to 8 (large basic proteins), while the second subfamily includes CKs from 9 to 19 (low molecular weight) [19]. The low weight CKs are expressed in glandular and simple epithelia, whereas the high weight CKs are expressed in squamous stratified keratinized epithelium $(18,19,21]$.

Moreover, the matrix metalloproteinase (MMP) family has been shown to be greatly involved, in particular MMP-9 and gelatinase B, in the breakdown of extracellular matrix (ECM) both in physiological and pathological processes. MMP-9 degrades types IV and $\mathrm{V}$ collagens and is essential for bone resorption through the osteoclastic activation by removing the collagen from the bone surface before demineralization starts [23].

The present study using immunohistochemical (IHC) methods, sheds light on the possible role of apoptosis and the histological origin of PAL and EGL, through studying factors affecting tissue homeostasis: apoptosis-related factors, cell proliferation, extracellular matrix, and differential cytokeratin expression.

\section{Material and methods}

Patients and tissue preparation. Between January 2009 and December 2011, 55 (30 with PAL and 25 with EGL) consecutive patients, were selected following informed consent and authorization from the local ethics committee. They were selected for the study based on clinical and radiological exams. The PAL patients had a severe grade of periapical lesions and tooth decay with exposure of the pulp chamber. They reached the clinic untreated for a very long time, usually, more then 12 months. PAL and EGL biopsies were surgically extracted and fixed in $10 \%$ buffered formalin for at least 24 hours and then processed in paraffin for routine light microscopy and stained with hematoxylin and eosin (HE) to study the histology and confirm diagnosis. Ten PAL and ten EGL biopsies, which were the least traumatized, were further processed for immunohistochemistry. Serial $8 \mu \mathrm{m}$ paraffin sections were prepared, 8 for treatment with primary antibodies and 3 negative controls in which primary antibody was omitted, for each type of antibodies. The primary antibodies against cytokeratin (CKs) 1, 5, 8, 10 and 14; caspase-3, caspase-9, metalloproteinases-9 (MMP-9) and PCNA were used for IHC technique using the detection kits En Vision + System-HRP with AEC as substrate (Dako, Italy). Apoptosis was detected using TUNEL assay.

Immunohistochemistry. The serial sections were cut on Leica microtome RM2145, dried overnight at $37^{\circ} \mathrm{C}$, and then stored at room temperature. The day after, the slides were dewaxed and rehydrated by sequential immersion in a graded series of alcohols and transferred into water for five minutes. To inhibit any endogenous peroxidase activity the slides were treated for five minutes with Peroxidase Block in hydrated incubation enclosure at room temperature. Subsequently, the slides were transferred into PBS ( $\mathrm{Na} 2 \mathrm{HPO} 4, \mathrm{KH} 2 \mathrm{PO} 4, \mathrm{KCl}, \mathrm{NaCl} \mathrm{pH}$ 7.4-7.6) at room temperature. The following protocol was realized using the kit En-Vision + System HRP with AEC as substrate (Dako). After rinsing with PBS, the sections were incubated overnight at $4^{\circ} \mathrm{C}$ with monoclonal anti-CK1, -5, -8, -10 and -14 (Santa Cruz Biotechnology, Inc), diluted 1:100, polyclonal anti-MMP-9 (Chemicon International) diluted 1:100; poly- 
clonal anti-caspase-3 and -9 (Sigma, USA) diluted 1:100 and clonal anti-PCNA (Dako) diluted 1:50. After the incubation, any excess antibody was removed by washing with PBS for five minutes. The sections were then incubated with Peroxidase labeled polymer conjugated to goat anti-rabbit immunoglobulin in Tris- $\mathrm{HCl}$ buffer containing stabilizing protein and an antimicrobial agent. Unbound polymer was removed by washing (2X with PBS, 5 minutes each) and subsequently AEC chromogen in substrate buffer was then added for five minutes and the reaction was stopped in distilled water. After removing the slides from water one drop of aqueous mounting medium (Dako Faramount) was applied, and the sections were coverslipped.

Negative controls were performed by omission of primary antibody, and by incubating sections with antiserum saturated with homologous antigen.

Finally the sections were observed with Leica DMLB microscopy. Densitometric quantification of all antibodies studied expression was performed by image analysis using "Image Pro Plus" software (Adobe System Inc, San Jose, CA).

Image Analysis. The immunohistochemical specimens were examined using a Leica Laborlux S Microscope (Leica Microsystem GmbH Wetzlar, Germany) with a Nikon DSL2 photo digital system (Nikon Corp, Tokyo, Japan). Each sample was analyzed with a double-blind system by two different operators. Moreover, the results were compared to an image analysis obtained from digital TIF files acquired with the multispectral system $(24,25)$. To apply this method, we made sequential shots using CoKin (Cokin SAS, Rungis Cedex, France) filters to obtain all the different color spectra. Adobe Photoshop CS4 extended (Adobe Systems Inc, San Jose, CA) was used to elaborate images $(26,27)$. Choosing the spectrum related to AEC, we converted the image color profile from RGB to CMYK. The yellow channel was selected based on the literature data which indicated that it has the best linear response to color intensity and thus to protein presence, range from 0 to 255 gray tone ( $Y$ in graphics) (28). The quantification of colorimetric staining was evaluated by scoring with values from $1+$ to $5+$, in 10 randomly selected areas, total of 100 positively stained cells [28-30].

TUNEL assay. Terminal deoxynucleotidyl transferase dUTP nick end labeling (TUNEL) reactions were performed as described by Song et al. [31], in serial sections dewaxed and washed twice with PBS. After the incubation with Proteinase K (Sigma) for 15 minutes at room temperature, the sections were washed twice with distilled water and incubated with $3 \%$ hydrogen peroxide in PBS for 5 minutes. The ApopTag Peroxidase In Situ Apoptosis Detection kit (Millipore, Cat. No. S7100) was used to label the free 3'-OH fragment DNA ends in situ with chemically labeled and unlabeled nucleotides (TdT-mediated). DNA fragments were labeled with the digoxigenin-nucleotide and allowed, for 30 minutes in humidified chamber at room temperature, to bind an anti-digoxigenin antibody conjugated to a peroxides reported molecule. Such binding was revealed using DAB as a substrate. Finally, the specimens were washed with distilled water and mounted under a cover slip in an Aqueous Mounting Medium (Dako). Photography was performed by Leica Laborlux S Microscope with a Nikon DSL2 photo digital system.

\section{Results}

The expression of all tested cytokeratins was found in PAL. CK1, 5, 8 and 10 showed a high degree of expression while CK14 was less than half of the rest and only expressed in the basal epithelial layer to the intensity almost half the other cytokines (Figures 1 and 4). In addition, the polymorph nuclear cells and plasma cells as well as the epithelium were very well stained for MMP-9 (Figures 2 and 4C). The TUNEL staining was also intense in restricted areas of the epithelium (Figures 2 and 4D). Moreover, there was a moderate but clear and distinct positivity of PCNA in the basal layer indicating the simultaneous presence of cell proliferation and apoptosis. The expression of caspase- 3 and -9 was found in PAL and EGL, however, it was less intense in PAL than in EGL (Figures 2 and 4 A, B).

In EGL and PAL the cytokeratins CK1, 5 and 8 were also intensely stained in the various layers of the specimen, with higher expression in EGL than PAL (Figure 3). The CK8 was strongly expressed in the epithelial spinal layer (Figures 1 and $3 \mathrm{H}$ ), while the CK10 was moderately expressed if compared with PAL (Figures 1 and 3I). On the other hand, the CK14 depicted very intense expression almost 5 times more than that of PAL. It was mostly localized in the basal and suprabasal epithelial layers, indicating the high rate of proliferation and differentiation of the cells in the basal layer (Figures 1 and 3J). With respect to caspase-3 and -9, both were highly expressed in both PAL and EGL, however, with higher intensity in EGL. The caspases -3 and -9 were highly expressed in the basal and other epithelial layers (Figures 2 and 4F, J). Concerning the MMP-9, its expression was also intense in the basal layer while it decreased and faded away in the upper surface granular and lucidum layers of epithelium. In addition, the MMP-9 was extensively and more significantly expressed in PAL compared to EGL. As for the TUNEL assay, the reaction was moderately expressed in various epithelial layers (Figures 2 and 4I) with higher expression in PAL as compared to EGL, similarly to the MMP-9 expression. On the other hand, the PCNA was strongly expressed in the basal layer of epithelium (Figures 2 

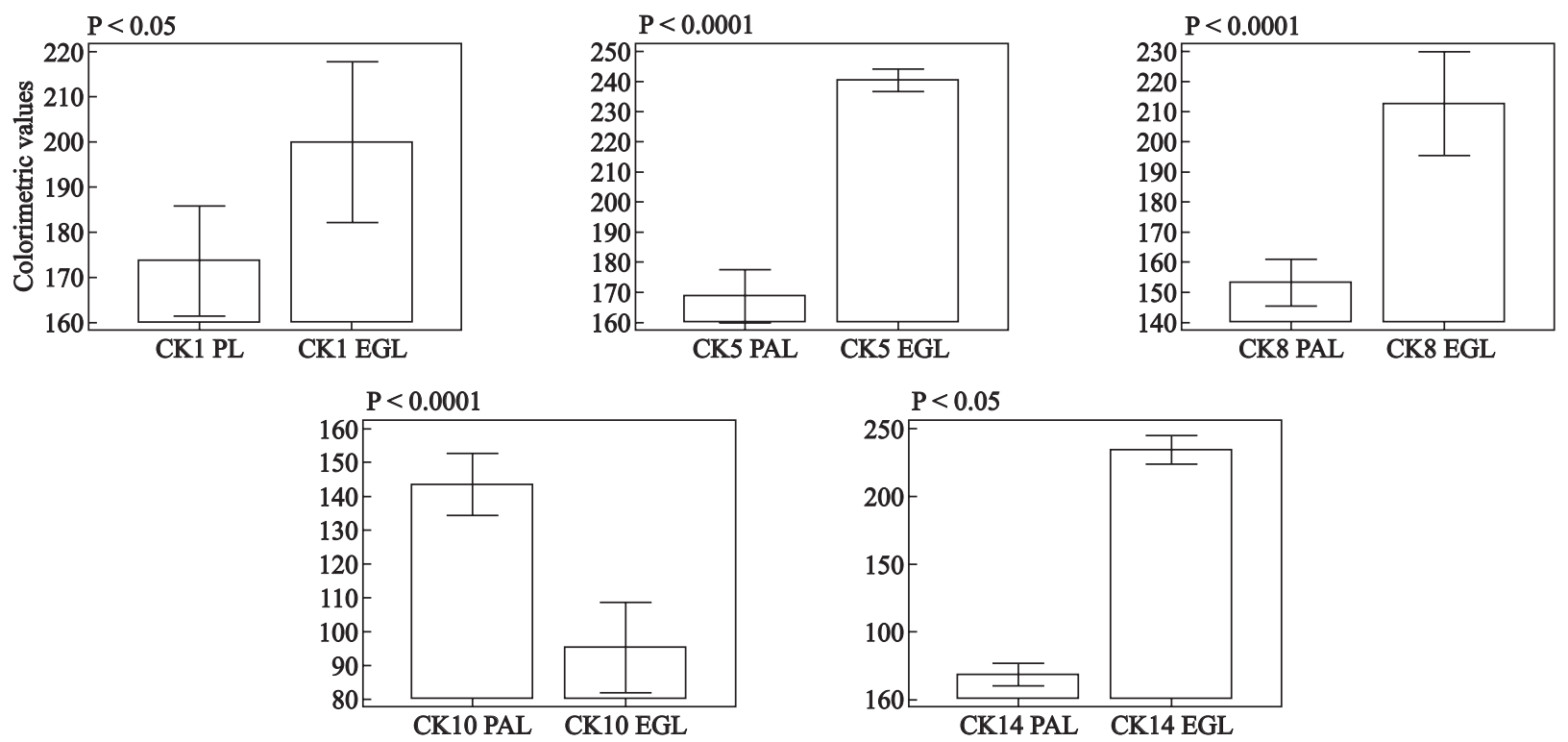

Figure 1. Comparison of the expression of various cytokeratins by immunohistochemistry in periapical (PAL) and epithelial gingival lesions (EGL). Y axis: the intensity of IHC staining was measured as described in Methods, bars represent means and whiskers: $95 \%$ CI. t-Test for independent samples: data show highly significant differences between PAL and EGL
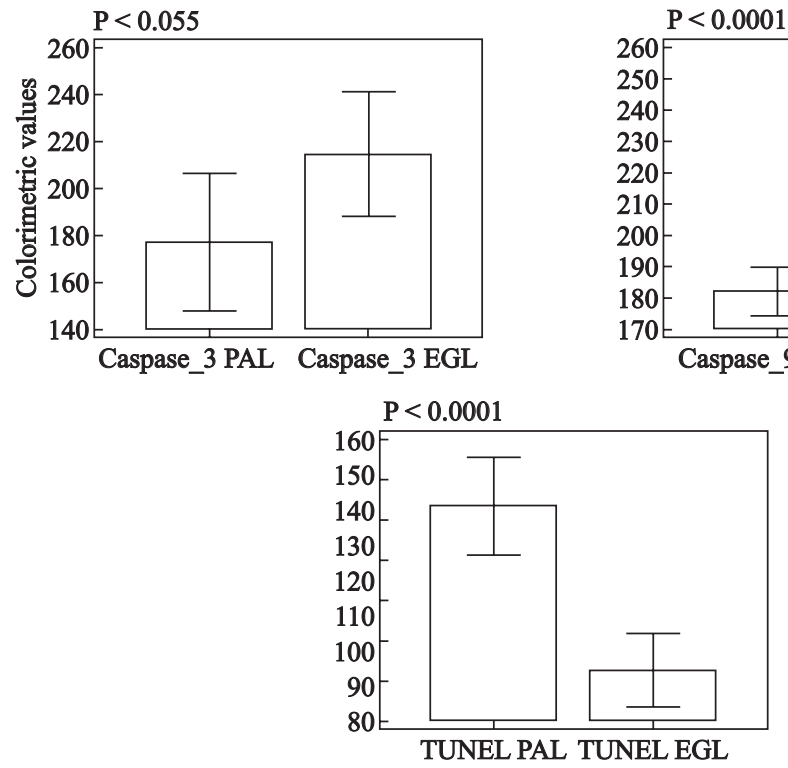
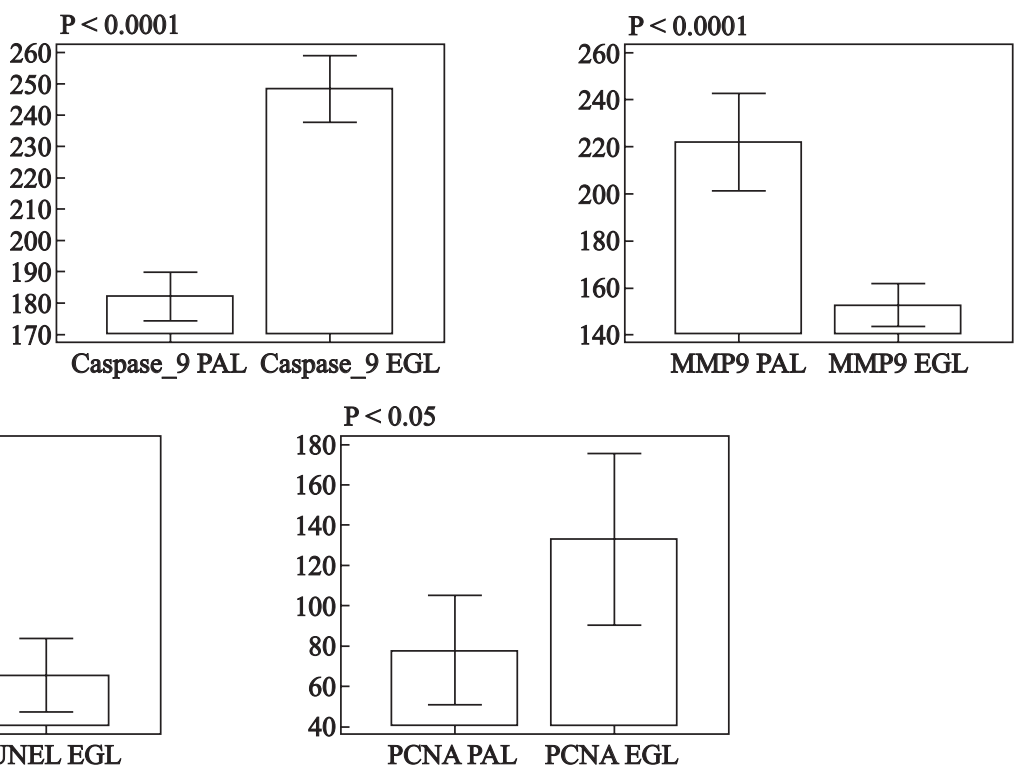

Figure 2. Comparison of the expression of caspase-3, caspase-9, MMP-9, and PCNA as well as apoptosis rate by TUNEL method by immunohistochemistry in periapical (PAL) and epithelial gingival lesions (EGL). Y axis: the intensity of IHC staining was measured as described in Methods, bars represent means and whiskers: $95 \%$ CI. t-Test for independent samples: data show highly significant differences between PAL and EGL with the exception of the caspase-3 expression

and $4 \mathrm{~J}$ ), and significantly more in the EGL, indicating a more active cell proliferation.

\section{Discussion}

The results indicated that apoptosis-related factors like caspases-3 and -9 were increased in the inflam- matory tissue of PAL and EGL very much like in the marginal periodontal legions. These inducers of apoptosis might have accelerated the process of apoptosis [32]. Actually, caspase- 3 and -9 were well expressed in the suprabasal and surrounding epithelial layers of both PAL and EGL. On the other hand, cytokeratins, markers for epithelial differentiation, as expected, 

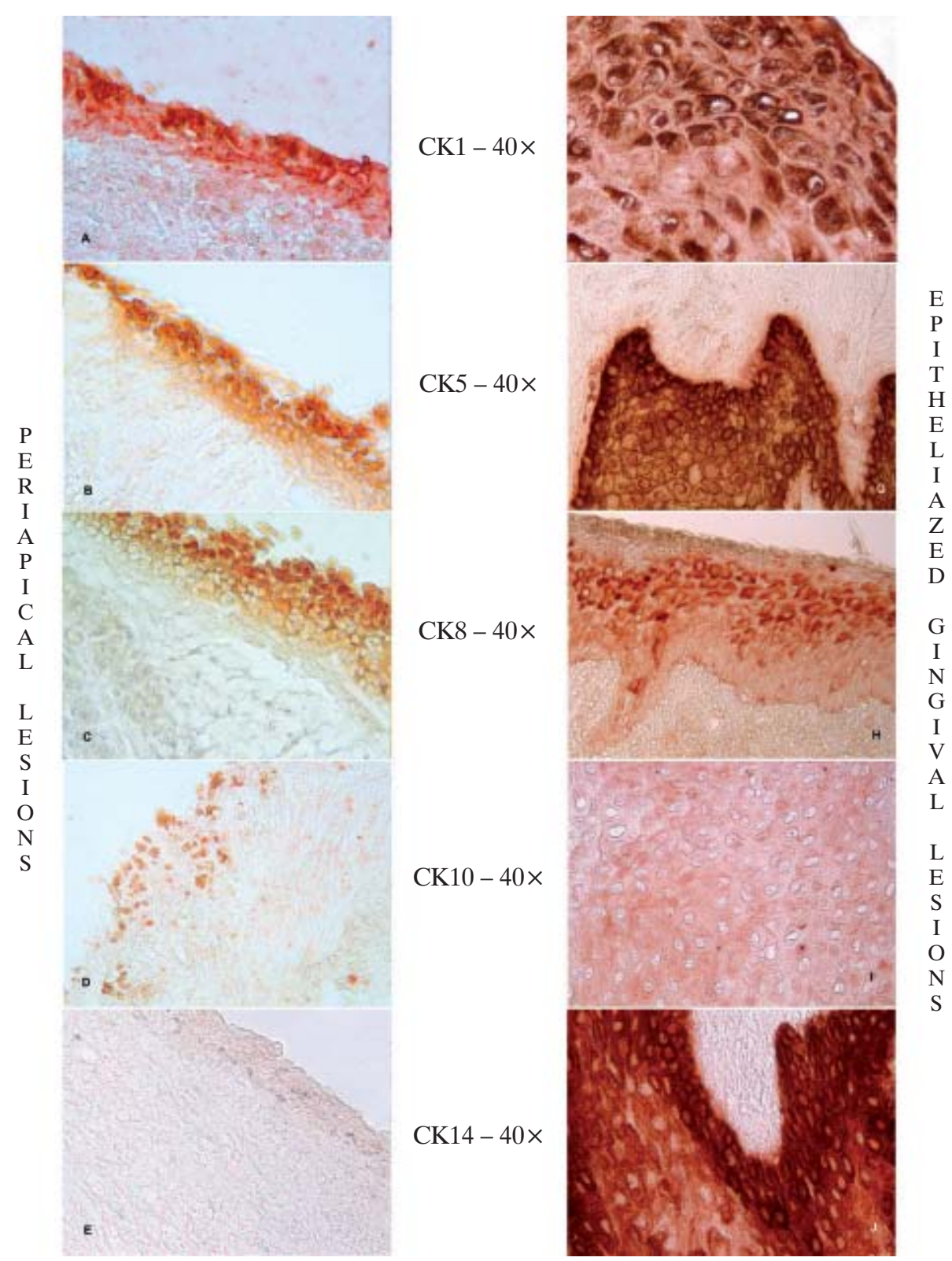

Figure 3. Immunostaining of cytokeratins CK1, 5, 8, 10 and 14 in PAL (left panel: A, B, C, D, E) and EGL (right panel: F, G, H, I, J). For details see Methods. Objective magnification $40 \times$.

were intensely stained in the epithelial layers, particularly, the basal and suprabasal layers. The CK1, CK5 and CK8, low molecular weight cytokeratins and CK14 (large molecular weight) were significantly more expressed in EGL compared to PAL. Such cytokeratins' expression was associated with stronger expression of PCNA in the basal layer, an indicator of increased rate of cell divisions and proliferation in EGL compared to PAL.

Furthermore, apoptosis expressed by TUNEL in PAL was restricted mostly to epithelial cells, while it was more diffused in in EGL. All these parameters indicated clearly that epithelial cells in both PAL and EGL were undergoing apoptosis with a relatively higher rate in EGL compared to PAL. Such results suggest that the epithelial proliferation and differentiation was being balanced to variable extents by apoptosis [32]. The very limited expression of most apoptotic factors such as caspase- 3 and -9 in the lining epithelium of PAL was consistent with a similarly restricted and lower proliferation and differentiation $[2,14,15]$.

The obtained data clearly suggest that apoptosisrelated factors and apoptotic reactions were involved 


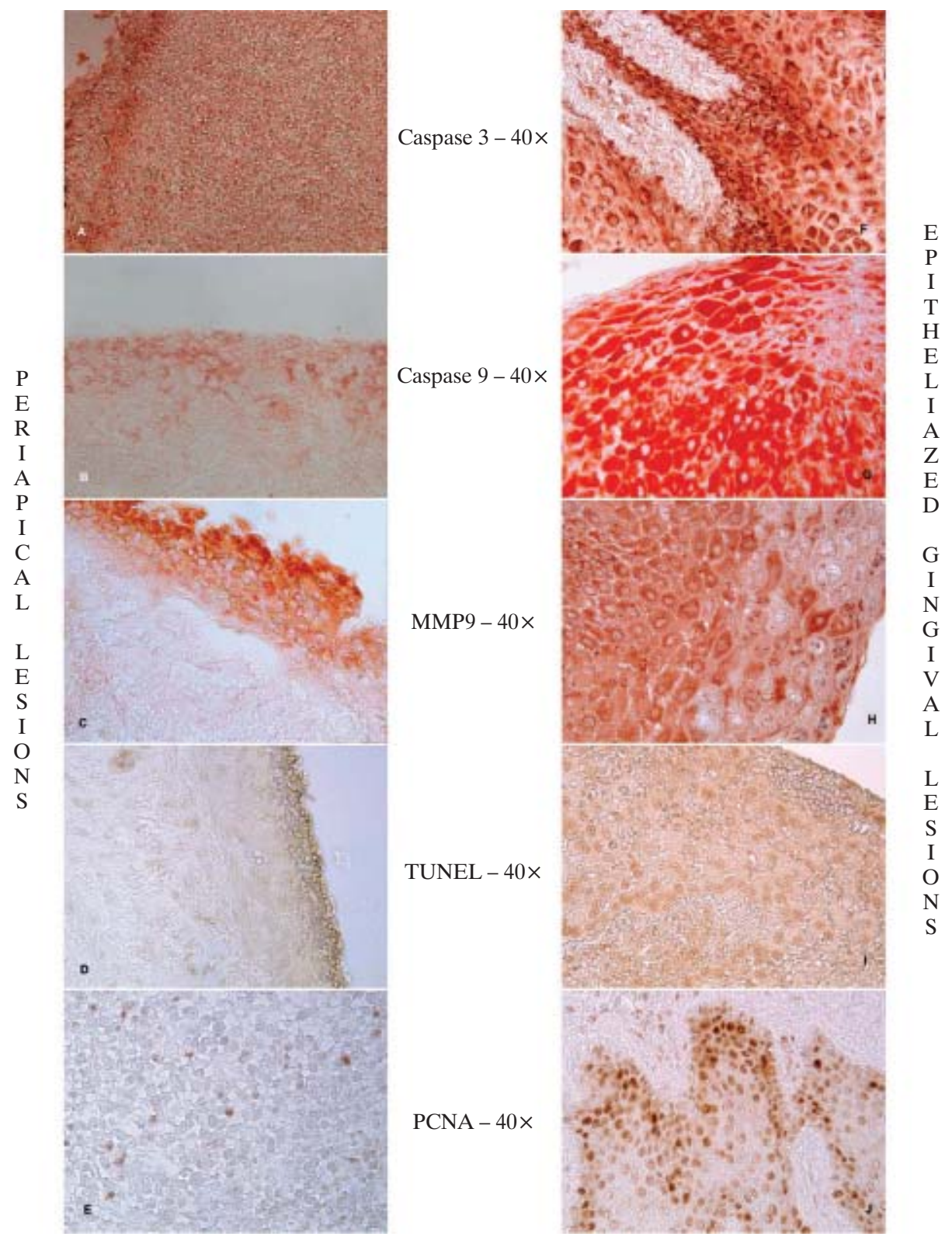

Figure 4. Immunostaining of caspase-3 and -9, MMP-9 and PCNA as well apoptosis detection by TUNEL method in PAL (left panel: A, B, C, D, E) and EGL (right panel: F, G, H, I, J). For details see Methods. Objective magnification $40 \times$.

in the pathophysiologic processes of PAL and EGL to variable extents. It is also likely that such involvement may be affected by the location and the degeneration of cell nuclei or the degree of inflammation, which was usually greater in the studied EGL samples than in PAL. There was obviously a good correlation between PCNA activity, caspases, and MMP-9 activities. Thus, it seems that the extent of inflammatory lesions was dependent on the balance between epithelial cell proliferation and cell death as shown for radicular and dentigeous cysts [33].
Apoptosis depends on the activity of caspases, and expression of caspase- 3 is considered essential for the activation of apoptosis. It was reported that an increase in caspase- 3 expression could be reflected in up-regulation of both apoptosis and proliferation of cyst lining epithelium [34]. An activated caspase-3 could have hydrolyzed various cellular proteins which could led to morphological changes of cells and organelles $[14,15]$. In our study we found that expression of caspases was higher relative to the TUNEL assay results. This difference resulted probably from the 
fact that degradation of cells and their content did not reach the stage of DNA fragmentation. Actually, the reported data in this article, showed that apoptotic parameters were overexpressed and were involved in the inflammatory processes; they could have played a role in the activation and proliferation of epithelium, leading to the progression of PAL and EGL.

In summary, the different, expression of caspase3 and caspase-9, MMP-9 and PCNA, as assessed by immunohistochemistry, in PAL and EGL suggests the involvement of apoptosis in the pathomechanisms of these two pathologies.

\section{References}

1. Leonardi R, Caltabiano M, Pagano M, Pezzuto V, Loreto C, Palestro G. Detection of vascular endothelial growth factor/vascular permeability factor in periapical lesions. J Endod. 2003;29:180-183.

2. Suzuki T, Kumamoto H, Kunimori K, Ooya K. Immunohistochemical analysis of apoptosis-related factors in lining epithelium of radicular cysts. J Oral Pathol Med. 2005;34:46-52.

3. Fujita Y, Ito H, Sekino S, Numabe Y. Correlations between pentraxin 3 or cytokine levels in gingival crevicular fluid and clinical parameters of chronic periodontitis. Odontology. 2011;100:215-221.

4. Nagata S. Fas ligand-induced apoptosis. Annu Rev Genet. 1999; 33:29-55.

5. Cappello F, Bellafiore M, Palma A, Bucchieri F. Defective apoptosis and tumorigenesis: role of p53 mutation and Fas/FasL system dysregulation. Eur J Histochem. 2002;46:199-208.

6. Tuan RS. Role of adult stem/progenitor cells in osseointegration and implant loosening. Int J Oral Maxillofac Implants. 2011;26 Suppl:50-62; discussion 63-9.

7. Mak BC, Wang Q, Laschinger C, Lee W, Ron D, Harding HP, Kaufman RJ, Scheuner D, Austin RC, McCulloch CA. Novel function of PERK as a mediator of force-induced apoptosis. J Biol Chem. 2008;283:23462-235472.

8. Bellmann K, Charette SJ, Nadeau PJ, Poirier DJ, Loranger A Landry J. The mechanism whereby heat shock induces apoptosis depends on the innate sensitivity of cells to stress. Cell Stress Chaperones. 2010;15:101-113.

9. Li P, Nijhawan D, Budihardjo I, Srinivasula SM, Ahmad M, Alnemri ES, Wang X. Cytochrome c and dATP-dependent formation of Apaf-1/caspase- 9 complex initiates an apoptotic protease cascade. Cell. 1997;14:479-489.

10. Hajra KM, Liu JR. Apoptosome dysfunction in human cancer. Apoptosis. 2004;9:691-704.

11. Gupta S, Knowlton AA. Cytosolic heat shock protein 60, hypoxia, and apoptosis. Circulation. 2002;106:2727-2733.

12. Cappello F, David S, Peri G, Farina F, Conway de Macario E, Macario AJ, Zummo G. Hsp60: molecular anatomy and role in colorectal cancer diagnosis and treatment. Front Biosci. (Schol Ed). 2011;1:341-351.

13. Samali A, Cai J, Zhivotovsky B, Jones DP, Orrenius SPresence of a pre-apoptotic complex of pro-caspase-3, Hsp60 and Hsp10 in the mitochondrial fraction of jurkat cells. EMBO J. 1999;18: 2040-2048.

14. Xanthoudakis S, Roy S, Rasper D, Hennessey T, Aubin Y, Cassady R, Tawa P, Ruel R, Rosen A, Nicholson DW. Hsp60 accelerates the maturation of pro-caspase- 3 by upstream activator proteases during apoptosis. EMBOJ. 1999 15;18:2049-2056.

15. Chandra D, Choy G, Tang DG. Cytosolic accumulation of HSP60 during apoptosis with or without apparent mitochondrial release: evidence that its pro-apoptotic or pro-survival functions involve differential interactions with caspase-3. J Biol Chem. 2007;282: 31289-31301.
16. Cappello F, Di Stefano A, David S, Rappa F, Anzalone R, La Rocca G, D'Anna SE, Magno F, Donner CF, Balbi B, Zummo G. Hsp60 and Hsp10 down-regulation predicts bronchial epithelial carcinogenesis in smokers with chronic obstructive pulmonary disease. Cancer. 2006;107:2417-2424.

17. Gregory CD, Pound JD. Cell death in the neighbourhood: direct microenvironmental effects of apoptosis in normal and neoplastic tissues. J Pathol. 2011;223:177-194.

18. García CC, Diago MP, Mira BG, Sebastián JV, Sempere FV. Expression of cytokeratins in epithelialized periapical lesions. Oral Surg Oral Med Oral Pathol Oral Radiol Endod. 2009;107:e43-46.

19. García CC, Sempere FV, Diago MP, Bowen EM. The post-endodontic periapical lesion: histologic and etiopathogenic aspects. Med Oral Patol Oral Cir Bucal. 2007;12:E585-590.

20. Sawaf MH, Ouhayoun JP, Forest NCytokeratin profiles in oral epithelial: a review and a new classification. J Biol Buccale. 1991; 19:187-198.

21. Marton IJ, Kiss C. Characterization of inflammatory cell infiltrate in dental periapical lesions. Int Endod J. 1993;26:131-136.

22. Moll R, Franke WW, Schiller DL, Geiger B, Krepler R. The catalog of human cytokeratins: patterns of expression in normal epithelia, tumors and cultured cells. Cell. 1982;31:11-24.

23. Carneiro E, Menezes R, Garlet GP, Garcia RB, Bramante CM, Figueira R, Sogayar M, Granjeiro JM. Expression analysis of matrix metalloproteinase-9 in epithelialized and nonepithelialized apical periodontitis lesions. Oral Surg Oral Med Oral Pathol Oral Radiol Endod. 2009;107:127-132.

24. Walker RA. Quantification of immunohistochemistry — issues concerning methods, utility and semiquantitative assessment I. Histopathology. 2006;49:406-410.

25. Taylor CR, Levenson RM. Quantification of immunohistochemistry - issues concerning methods, utility and semiquantitative assessment II. Histopathology. 2006;49:411-424.

26. Lehr HA, Mankoff DA, Corwin D, Santeusanio G, Gown AM.Application of photoshop-based image analysis to quantification of hormone receptor expression in breast cancer. $J$ Histochem Cytochem. 1997;45:1559-1565.

27. Kirkeby S, Thomsen CE. Quantitative immunohistochemistry of fluorescence labelled probes using low-cost software. J Immunol Methods. 2005;301:102-113.

28. Pham NA, Morrison A, Schwock J, Aviel-Ronen S, Iakovlev V, Tsao MS, Ho J, Hedley DW. Quantitative image analysis of immunohistochemical stains using a CMYK color model. Diagn Pathol. 2007;27:2-8.

29. Skaland I, Ovestad I, Janssen EA, Klos J, Kjellevold KH, Helliesen T, Baak JP. Digital image analysis improves the quality of subjective HER-2 expression scoring in breast cancer. Appl Immunohistochem Mol Morphol. 2008;16:185-190.

30. Skaland I, Rvestad I, Janssen EA, Klos J, Kjellevold KH, Helliesen T, Baak JP. Comparing subjective and digital image analysis HER2/neu expression scores with conventional and modified FISH scores in breast cancer. J Clin Pathol. 2008;61:68-71.

31. Pritlove-Carson S, Charlesworth S, Morgan PR, Palmer RM. Cytokeratin phenotypes at the dento-gingival junction in relative health and inflammation, in smokers and nonsmokers. Oral Dis. 1997:3:19-24

32. Suzuki T, Kumamoto H, Ooya K, Motegi K .Expression of inducible nitric oxide synthase and heat shock proteins in periapical inflammatory lesions. J Oral Pathol Med. 2002;31:488-493.

33. Martins CA, Rivero ER, Dufloth RM, Figueiredo CP, Vieira DS. Immunohistochemical detection of factors related to cellular proliferation and apoptosis in radicular and dentigerous cysts. $J E n$ dod. 2011;37:36-39.

34. Kimi K, Kumamoto H, Ooya K, Motegi K. Immunohistochemical analysis of cell-cycle- and apoptosis-related factors in lining epithelium of odontogenic keratocysts. J Oral Pathol Med. 2001;30:434-442. 